\title{
A Deterministic Simulation Model for Sojourn Time in Urban Cells with Square Street Geometry
}

\author{
Georgios S. Paschos, ${ }^{1}$ Ioannis G. Tsoulos, ${ }^{2}$ Efstathios D. Vagenas, ${ }^{3}$ \\ Stavros A. Kotsopoulos, ${ }^{3}$ and George K. Karagiannidis ${ }^{4}$ \\ ${ }^{1}$ National Research Center, VTT, P.O. Box 1000, Vuoremiehentie 5, 02150 Espoo, Finland
${ }^{2}$ Department of Computer Science, University of Ioannina, P.O. Box 1186, 45110 Ioannina, Greece
${ }^{3}$ Wireless Telecommunications Laboratory, Department of Electrical and Computer Engineering, University of Patras,
26500 Kato Kastritsi, Greece
${ }^{4}$ Division of Telecommunications, Department of Electrical and Computer Engineering, Aristotle University of Thessaloniki,
Serres, 54124 Veria, Greece
}

Correspondence should be addressed to Georgios S. Paschos, gpasxos@ee.upatras.gr

Received 25 May 2007; Revised 31 January 2008; Accepted 8 April 2008

Recommended by Chung-Ju Chang

A deterministic simulation model for the calculation of sojourn time statistics is proposed. The model is designed for urban cells taking into account city movement in street pattern, traffic lights, and crossings. Sojourn time and remaining sojourn time are assumed to be random variables that follow a gamma distribution. The parameters of gamma are found for a range of the chosen cell parameters (road length, probability of stopping at a crossing and cell radius) using Monte Carlo simulation. Then, the values are fitted to the empirical model using genetic algorithms with grammatical evolution. The model can be used to calculate sojourn time statistical parameters for any urban circular cell with square street pattern.

Copyright ( $) 2008$ Georgios S. Paschos et al. This is an open access article distributed under the Creative Commons Attribution License, which permits unrestricted use, distribution, and reproduction in any medium, provided the original work is properly cited.

\section{Introduction}

Sojourn time (ST) is defined as the time spent by a vehicle user inside a cell while using a communication channel. It is originally defined for a handover call, in which the vehicle starts its journey at the edge of the cell. For a new call, where the vehicle starts from a random position inside the cell, the channel holding time is called remaining sojourn time (RST). These two random variables, obviously, depend on characteristics of the cell area. In case of an urban cell, the important characteristics are the road pattern, the traffic lights, the crossings, and the city traffic.

Sojourn time statistics have been investigated in the past due to their usability in teletraffic analysis and cell planning. In [1], it is shown that sojourn time affects the handover ratio and, therefore, it is an important characteristic of a cellular network regarding the resource management. In [1], also a simple analytical model is presented, where movement in a straight line is considered. The results in [1] show an exponential-like behavior. In [2], a more realistic approach is chosen where the vehicles move in a series of vectors and perform random stops in between. In this case, the result is a statistical model that describes a specific cell environment. An extension to [2] is found in [3], where the same approach is enhanced with more complex stochastic models to better describe velocity, street length, and probabilities of turning. An important addition of [3] is that average step velocity is measured and the data are fit in a weighted sum of Rice and Gauss distribution. An analytical approach of the statistical model of [2,3] is found in [4]. Other previous works, relative to the present, can be found in [5-7].

However, applying all the above models in a real-life cell remains challenging since all of them demand extra effort for each new cell scenario, that is, because they fit measurements or simulations of specific cells to their models, but they do not provide any means of predicting the behavior of ST and RST in an arbitrary cell. Moreover, measurements of ST and RST are particularly time consuming and tedious. Channel holding time measurements are easier to obtain from a mobile operator as in [8], but in this case ST and RST 
information is mixed and the cell is assumed to be operating (information not available in design state). In this paper, we propose an empirical model that can be directly applied to any city cell with respect to some assumptions for ST and RST prediction.

The proposed model is a statistical model which can be seen as an extension of [9]. ST and RST are assumed to follow a gamma distribution as in [2-4, 9]. However, this model connects a set of cell parameters with the gamma distribution parameters $a$ and $b$. A road map can be used to extract the cell parameters for any cell that fulfills certain requirements, and then to use them as inputs to the model. A measurements case study carried through in a real cell shows a satisfactory model performance.

Apart from the empirical formulas for computing ST and RST statistics, this paper provides some novel ideas on this topic. The use of fixed velocity distribution is proposed, implying that the driver behavior is always the same in urban environments. Issues related to traffic are explained further. In comparison to [9], the cell radius parameter is added to provide solution to any cell size ranging from $100 \mathrm{~m}$ to $1 \mathrm{~km}$. In addition, the derivation of turning probability is elaborated to incorporate normal driver behavior. The fitting approach, that is also improved and combined with the above improvements, yields better approximation.

The mobility model is described in Section 2, and the empirical formulas are given in Section 3. A real cell study is found in Section 4, where the model is compared to measurements, and finally the paper is concluded in Section 5.

\section{The Proposed Deterministic Simulation Model}

In this section, the proposed model is described. The design process is analyzed and the necessary assumptions are explained.

\subsection{Model Design}

The model design procedure is described in Figure 1. An intuitive set of three cell parameters is chosen. These three parameters are the ones used to characterize an arbitrary cell, and they constitute the domain of the empirical functions produced by the model. For all possible values of these parameters, a Monte Carlo simulation is performed and the results are fitted to the gamma distribution. The gamma parameters $a$ and $b$ are then extracted with a median regression method. After the simulation loop, $a^{\mathrm{ST}}, a^{\mathrm{RST}}, b^{\mathrm{ST}}$, and $b^{\mathrm{RST}}$ data are available and they are used as inputs to a graph fitting procedure by means of a genetic algorithm with grammatical evolution. The final model is an expression of $a$ and $b$ in terms of the chosen parameters. Then, the distribution of ST and RST is obtained as

$$
f(x)=\frac{1}{b^{a} \Gamma(a)} x^{a-1} e^{-x / b}
$$

The chosen cell parameters are the probability of traffic $p_{\text {tr }}$, the normalized average block area $A_{\mathrm{b}}$, and the cell radius

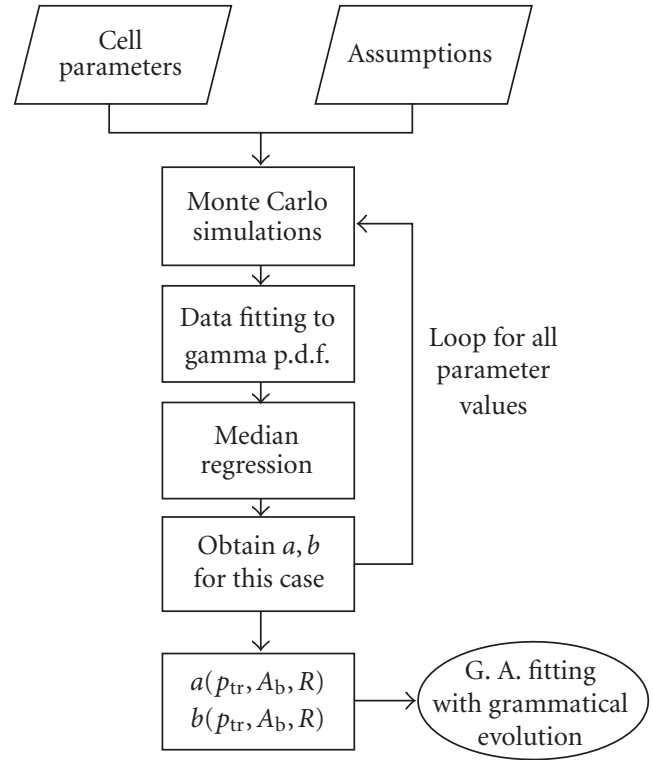

Figure 1: Logical diagram of the model design procedure.

$R$. The first parameter $p_{\mathrm{tr}}$ is the probability that a vehicle user stops for a pause time in an arbitrary crossing. For a set of crossings $X$ with $N^{X}$ elements which $N_{\text {tr }}^{X}$ of them have traffic lights, we write

$$
p_{\mathrm{tr}}^{X}=\frac{N_{\mathrm{tr}}^{X}+0.5\left(N^{X}-N_{\mathrm{tr}}^{X}\right)}{N^{X}} .
$$

The vehicle user faces three possibilities, making an average pause time at a traffic light crossing, making a pause time at a nontraffic light crossing coming from the minor road and making no pause time when coming from a major road. Every traffic light crossing is associated with an average delay and half of the priority crossings are associated with a deterministic delay. By using all the crossings in a cell $(X=C), p_{\mathrm{tr}}=p_{\mathrm{tr}}^{C}$ describes a cell where the movement is uniformly distributed among all roads. Another approach is to use the set $M$ that contains all the crossings of major roads that sustain the most important traffic load. This corresponds to transitional traffic in the cell. Assuming that the probability that an arbitrary user is in transitional state $(P$ (in transit $))$ is known, we get

$$
p_{\mathrm{tr}}=P(\text { in transit }) p_{\mathrm{tr}}^{M}+(1-P(\text { in transit })) p_{\mathrm{tr}}^{C} .
$$

The normalized average area of block, $A_{\mathrm{b}}$, can be used to extract the length of the roads. In case of square blocks

$$
d=\sqrt{A_{\mathrm{b}}}+\mathrm{rw} \approx \sqrt{A_{\mathrm{b}}},
$$

where $d$ is the edge, and rw is the road width. The value of $A_{\mathrm{b}}$, in case of nonidentical blocks, is computed by averaging which results in a possible error. The greater the square diversity, the greater the error is expected.

The third parameter is simply the cell radius, $R$. However, this parameter is necessary for the application of the model in an arbitrary cell. The inclusion of this parameter is an 


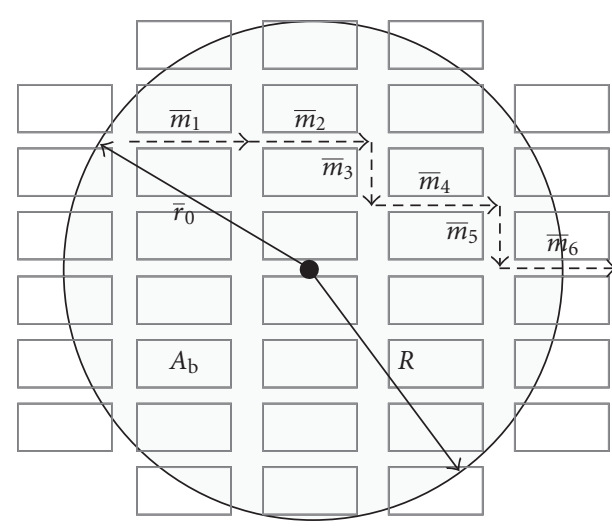

FIGURE 2: A random walk on a lattice example.

extension to [9]. Average values for $N^{X}, N_{\mathrm{tr}}^{X}$, rw, and $R$ can be found in a road map.

\subsection{Deterministic Laws of Mobility}

The random walk on a lattice used in the model is showcased in Figure 2. The position vector $\bar{r}$ for step $i$ will be

$$
\overline{r_{i}}=\overline{r_{0}}+\sum_{k=1}^{i} \overline{m_{k}}
$$

where $\overline{r_{0}}$ is the starting position, and $\left|\overline{m_{k}}\right|$ is the distance that a vehicle makes in step $k .\left|\overline{m_{k}}\right|$ is fixed and equal to $d$ in the context of square pattern. In every crossing, the vehicle makes a turn $\varphi_{i}=\arccos \left(\overline{m_{i-1}} \cdot \overline{m_{i}} /\left|\overline{m_{i-1}}\right|\left|\overline{m_{i}}\right|\right)$ which is a discrete random variable with $[0, \pi / 2,-\pi / 2]$ possible values. The vehicle exits the cell of radius $R$ for the first time at the $n$th step when $\left|\overline{r_{i}}\right|>R$ is true for $i=n$ but not true for all $i<n$. Assuming $u_{i}$ to be a positive Gaussian random variable that describes the average velocity of the vehicle in every step, the sojourn time for a random walk on a lattice exiting the cell of radius $R$ with $n$ steps will be

$$
\mathrm{st}=\sum_{i=1}^{n-1}\left(\frac{\left|\overline{m_{i}}\right|}{u_{i}}+\tau \cdot \operatorname{tr}_{i}\right)+\lambda \cdot\left(\frac{\left|\overline{m_{n}}\right|}{u_{n}}+\tau \cdot \operatorname{tr}_{n}\right)
$$

where $\operatorname{tr}_{i}$ is a binomial random variable with $p=p_{\mathrm{tr}}, \tau$ is an average delay in a traffic light or a stop sign, and $\lambda$ is the percentage of the last step which is inside the cell area. Using the law of cosines on the triangle, defined by $\overline{r_{n}}$ and the intersection of the circle and $\overline{m_{n}}$, we can calculate $\lambda$ from

$$
\left|\overline{m_{n}}\right|^{2} \lambda^{2}+\left(\left|\overline{r_{n}}\right|^{2}-\left|\overline{r_{n-1}}\right|^{2}-\left|\overline{m_{n}}\right|^{2}\right) \lambda=R^{2}-\left|\overline{r_{n}}\right|^{2} \text {. }
$$

Equation (7) has only one positive solution because $\left(\left|\overline{r_{n}}\right|^{2}-\left|\overline{r_{n-1}}\right|^{2}-\left|\overline{m_{n}}\right|^{2}\right)>0$. The above analysis is used in the simulation model and it corresponds to ST for $\left|\overline{r_{0}}\right|=R$ and RST for $\left|\overline{r_{0}}\right|<R$.

\subsection{Assumptions}

\subsubsection{Velocity}

Measurements in [3] have shown that average velocity in cities can be approximated by a weighted Gaussian and Rician distribution. In [9], however, it is proposed that this behavior is the result of the distortion of a Gaussian velocity caused by the pause times. This means that modeling the velocity as Gaussian and including probabilistic pause times at crossings produce a more realistic and intuitive model that can fit the measurements of [3].

In this paper, average velocity in every step is modeled as positive Gaussian with fixed mean and deviation $\left(\bar{u}_{i}=\right.$ $40 \mathrm{kmh}, \sigma_{u_{i}}=10 \mathrm{kmh}$ ). This implies that velocity is not a main characteristic of sojourn time in urban cells as opposed to other previous models referring to an arbitrary cell. The vehicle velocity in urban environment depends on the following factors:

(i) the velocity at which the driver would like to move,

(ii) the city regulations,

(iii) the crossings and the traffic lights,

(iv) the traffic.

Assuming that the driver behavior and the regulations are qualitatively the same in every city, the average velocity depends mostly on the cell shape and the expected traffic. The effect of traffic jams is not considered in this paper and, therefore, velocity is considered a positive Gaussian random variable with fixed parameters which is distorted by the delays in the crossings. This means that the model is valid for all city cells without extreme traffic. In case of extreme traffic, sojourn times are expected to be comparable to call duration times and the number of handovers is expected to be very small.

\subsubsection{Crossing Delay}

The crossing delay is $\tau$ with probability $p_{\text {tr }}$ and 0 with probability $1-p_{\operatorname{tr}}$ as described by the binomial random variable $\operatorname{tr}_{i}$. The value $\tau=10$ seconds is typically used as an average waiting time in a traffic light or a stop sign. Delay is 0 in case of a priority road crossing. It is expected that summing up many crossings with traffic lights and/or stop signs, the sum of waiting time divided by the number of such crossings, converges to the mean waiting time per crossing.

\subsubsection{Turning in The Crossings}

The formulation of turns has an important effect on the realism of the model. In bibliography $[3,9]$, it is proposed that the probable cases listed in descending order are $0,-\pi / 2$ (right) and $\pi / 2$ (left). Making a $\pi$ turn is considered highly improbable in a city environment. Moreover, conditional probabilities of turning are used to make the model more realistic. Turning left or right becomes more improbable after every turn in the same direction. This makes sure 
that a driver with a chosen destination is simulated much more times than one that roams around the cell in random directions. If conditional probabilities are neglected, the resulting model yields larger values on the average, like in [9]. The values used in our model are motivated by simulations and [3] $p_{\pi / 2}^{0}=0.135, p_{-\pi / 2}^{0}=0.165, p_{0}=1-p_{\pi / 2}^{k}-p_{-\pi / 2}^{l}$, and $p_{\pi / 2}^{k}=0.5 p_{\pi / 2}^{k-1}, p_{-\pi / 2}^{l}=0.5 p_{-\pi / 2}^{l-1}$, where $k$ and $l$ count the $\pi / 2$ and $-\pi / 2$ turns, respectively.

\subsection{Complexity Versus Accuracy}

Summarizing the previous subsections, the proposed model assumes vehicle movement in a square pattern urban cell with nonrush hour traffic. Moreover, it assumes that an arbitrary driver behaves in a standard way with respect to velocity and probability of turning in a crossing. Finally, the waiting time in a crossing is modeled the same in case of a traffic light (as an average) and in case of a nonpriority road (with a stop sign). It is expected that the accuracy of the model can be compromised when applied in a real life cell with many irregularities. The main advantage of the model is the simplicity with which it can be used. It should be used in the same way that radio propagation models for signal strength predictions are used.

\subsection{Genetic Algorithm with Grammatical Evolution}

The results of Monte Carlo simulations are fitted to a function using a genetic algorithm with grammatical evolution. Grammatical evolution is an evolutionary process that can create programs in an arbitrary language [10]. The production is performed using a mapping process governed by a grammar expressed in Backus Naur Form. The problem of data fitting can be formulated as given $N$ points and associated values $\left(x_{i}, y_{i}\right), i \in[1, N]$, with $x_{i} \in \mathbb{R}^{n}$, estimate a function $f: \mathbb{R}^{n} \rightarrow \mathbb{R}$ that minimizes the least squares error:

$$
E_{T}=\sum_{i=1}^{M}\left(f\left(x_{i}\right)-y_{i}\right)^{2} .
$$

The specific method takes as input the points $\left(x_{i}, y_{i}\right)$ and creates a functional form that minimizes the quantity in (8) through the procedure of grammatical evolution.

\section{Results}

The results are presented in Figures 3-6 for $R=100$ and $R=250$. Rate parameter $b$ has monotonous behavior, while the scale parameter $a$ is fluctuating in some cases. This fluctuation is justified in cases the actual distribution of sojourn time is changing shape. In such transition phase, the shape parameter is expected to be unstable.

From the figures, it is observed that greater street lengths $A_{\mathrm{b}}$, smaller probability $p_{\mathrm{tr}}$, and cell radius $R$ yield smaller $b$ and consequently smaller average sojourn times, which supports common reasoning.

The functions $a^{\mathrm{ST}}, a^{\mathrm{RST}}, b^{\mathrm{ST}}, b^{\mathrm{RST}}$ are produced by using the data from the deterministic simulation model described

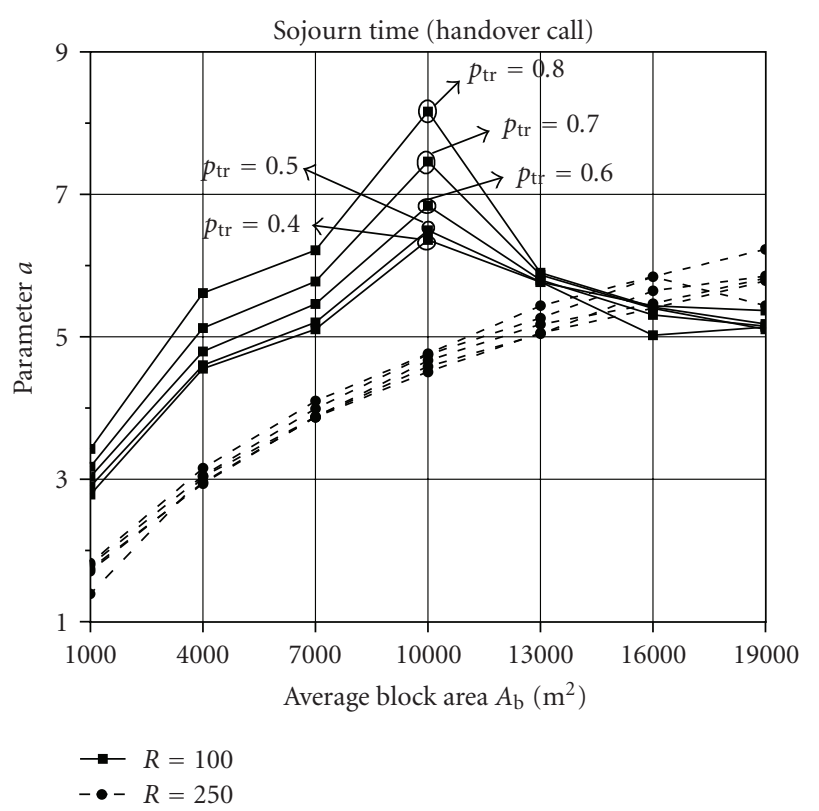

Figure 3: The function $a^{\mathrm{ST}}\left(p_{\mathrm{tr}}, A_{\mathrm{b}}, R\right)$ for $R=100$ and $R=250$.

in Section 2 and applying the method of fitting described in Section 2.5 [10]. For the chosen range of parameters values, there is a clear dependence of $a$ and $b$, on each of the parameters. This shows small information redundancy and supports the choice of parameters:

$$
\begin{aligned}
& a^{\mathrm{ST}}\left(p_{\mathrm{tr}}, A_{\mathrm{b}}, R\right) \\
& =2.49 \cdot 2.37^{\sin \left(\log \left(A_{\mathrm{b}}^{0.682707} / R\right)\right)}+\log \left(\log \left(\log \frac{6.99}{\cos p_{\mathrm{tr}}}+\sqrt{p_{\mathrm{tr}}}\right)\right), \\
& b^{\mathrm{ST}}\left(p_{\mathrm{tr}}, A_{\mathrm{b}}, R\right) \\
& \quad=\frac{R}{\sqrt{3 A_{\mathrm{b}} / R \cdot p_{\mathrm{tr}}+1.41772 / p_{\mathrm{tr}}+\cos \left(2.36 p_{\mathrm{tr}}\right)}}, \\
& \quad=\frac{\log \left(\log \left[R^{2} \log R / 433.77-A_{\mathrm{b}}^{2} \log p_{\mathrm{tr}} / 7814+R\right]\right)}{2}, \\
& a^{\mathrm{RST}}\left(p_{\mathrm{tr}}, A_{\mathrm{b}}, R\right) \\
& \quad=\frac{3.68638 \cdot R \sqrt{p_{\mathrm{tr}}}}{b^{\mathrm{RST}}\left(p_{\mathrm{tr}}, A_{\mathrm{b}}, R\right)} p_{\mathrm{tr}}\left(\log A_{\mathrm{b}}+1\right),
\end{aligned}
$$

where $\log$ is the natural logarithm. The mean least square error of fitting was found to be $e\left(a^{\mathrm{ST}}\right)=0.0315, e\left(b^{\mathrm{ST}}\right)=$ $0.0019, e\left(a^{\mathrm{RST}}\right)=0.4244$, and $e\left(b^{\mathrm{RST}}\right)=0.3794 .2000$ generations that were used in the fit process. The products $a^{\mathrm{ST}} \cdot b^{\mathrm{ST}}$ and $a^{\mathrm{RST}} \cdot b^{\mathrm{RST}}$ yield the expectation of ST and RST, respectively, while it is possible to calculate variance by $a^{\mathrm{ST}} \cdot\left[b^{\mathrm{ST}}\right]^{2}$ and $a^{\mathrm{RST}} \cdot\left[b^{\mathrm{RST}}\right]^{2}$. 


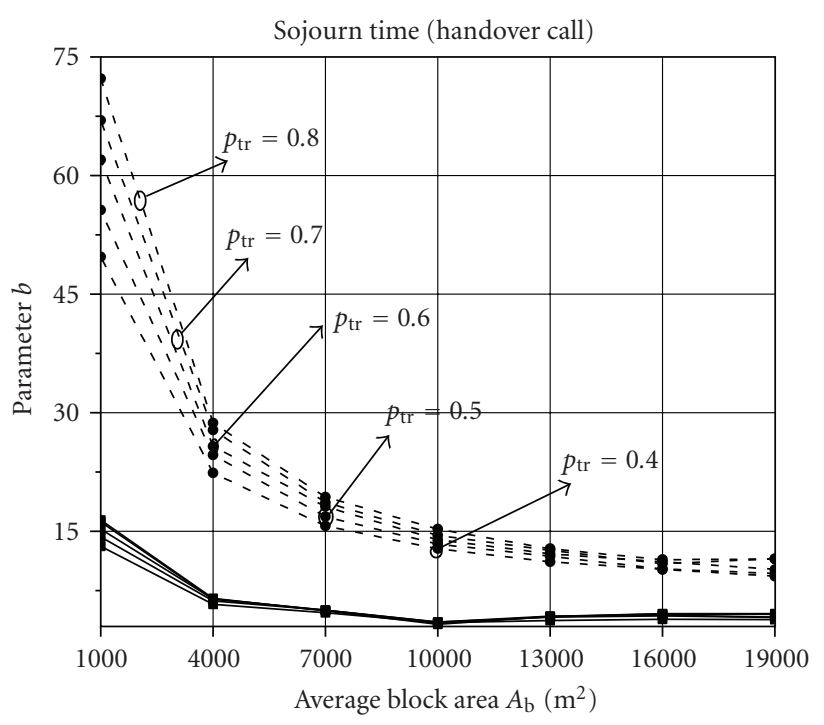

$\rightarrow R=100$

- - $R=250$

Figure 4: The function $b^{\mathrm{ST}}\left(p_{\mathrm{tr}}, A_{\mathrm{b}}, R\right)$ for $R=100$ and $R=250$.

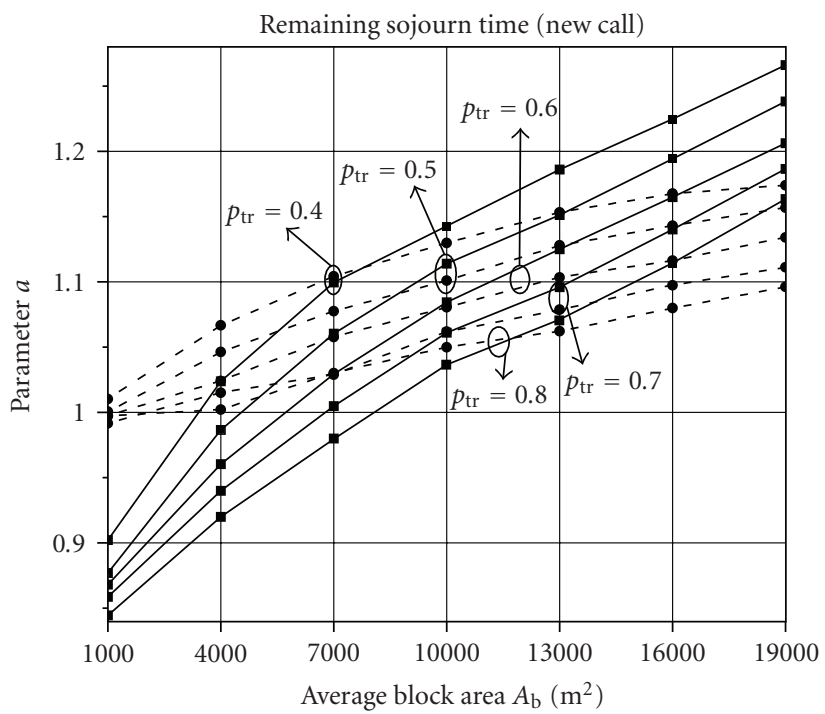

$\rightarrow-R=100$

Figure 5: The function $a^{\mathrm{RST}}\left(p_{\mathrm{tr}}, A_{\mathrm{b}}, R\right)$ for $R=100$ and $R=250$.

\section{Case Study in A real Cell}

A case study is presented for the cell of Figure 7. Using the road map, the values $A_{\mathrm{b}}=9070 \mathrm{~m}^{2}(95 \mathrm{~m} \times 95 \mathrm{~m})$ and $R=$ $300 \mathrm{~m}$ are extracted. For $p_{\mathrm{tr}}$, a traffic assumption is made. The $80 \%$ of the vehicles are assumed to use the major roads (yellow on the map) only and the rest are assumed to move randomly in the cell. Therefore, $p_{\mathrm{tr}}=0.8 \cdot p_{\mathrm{tr}}^{M}+0.2 \cdot p_{\mathrm{tr}}^{C}$, where

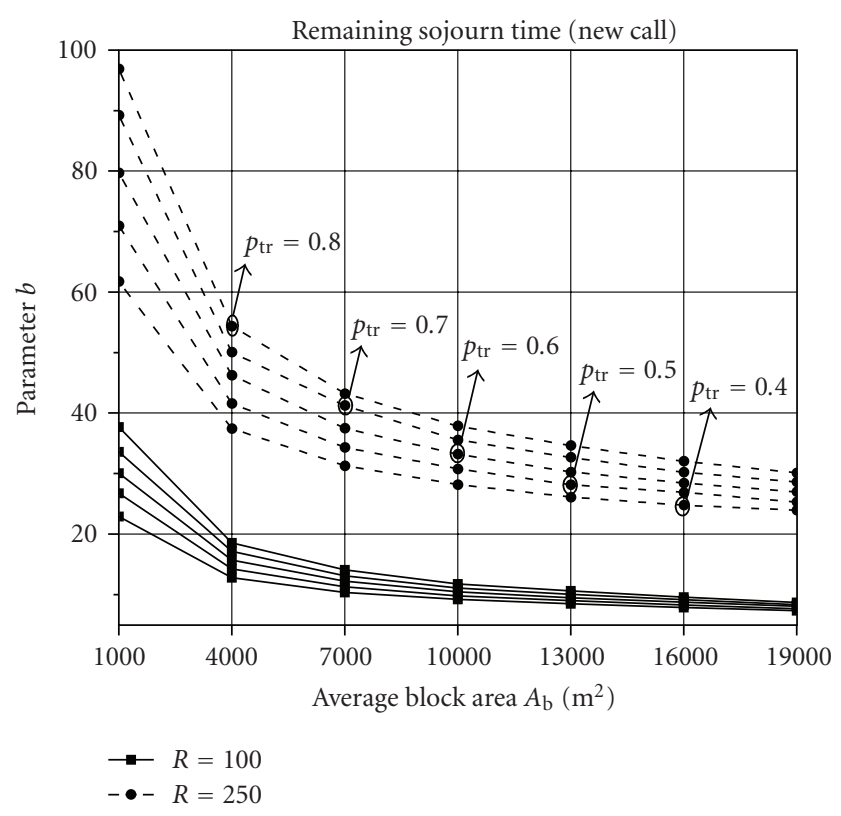

Figure 6: The function $b^{\mathrm{RST}}\left(p_{\mathrm{tr}}, A_{\mathrm{b}}, R\right)$ for $R=100$ and $R=250$.

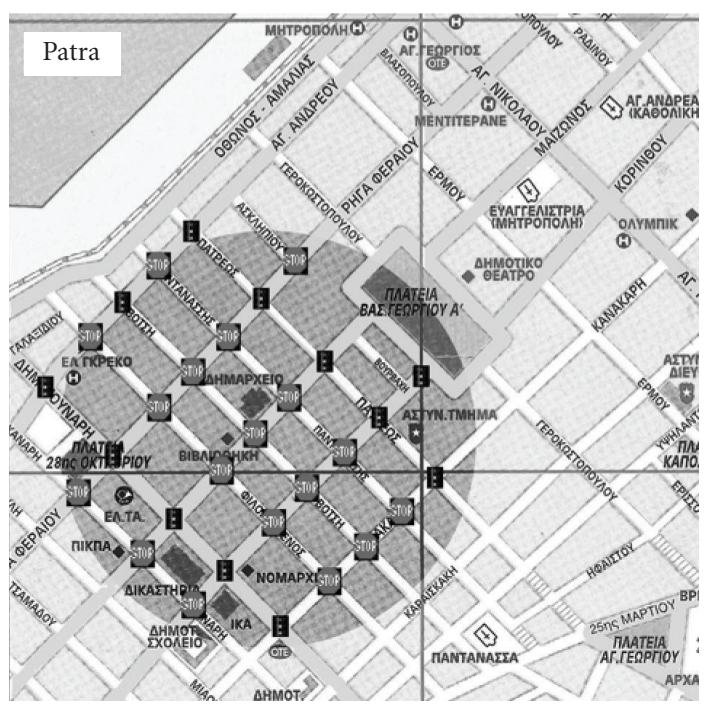

Figure 7: The cell in the city of Patras under test. Measurements took place in this cell.

$p_{\mathrm{tr}}^{M}=0.571$ and $p_{\mathrm{tr}}^{c}=0.7$ using (2). This yields the value $p_{\text {tr }}=0.597$.

Using $(9), a^{\mathrm{ST}}=3.88094, b^{\mathrm{ST}}=24.5184, a^{\mathrm{RST}}=$ 1.08972 , and $b^{\mathrm{RST}}=44.6443$ are calculated. These results are compared to measurements taken in the same area in nonrush hour. The measurements density is estimated using Epanechnichov kernel-based estimator with half the smoothing optimal bandwidth $\left(h_{\mathrm{os}} / 2\right)$, see [11]. In Figure 8, 


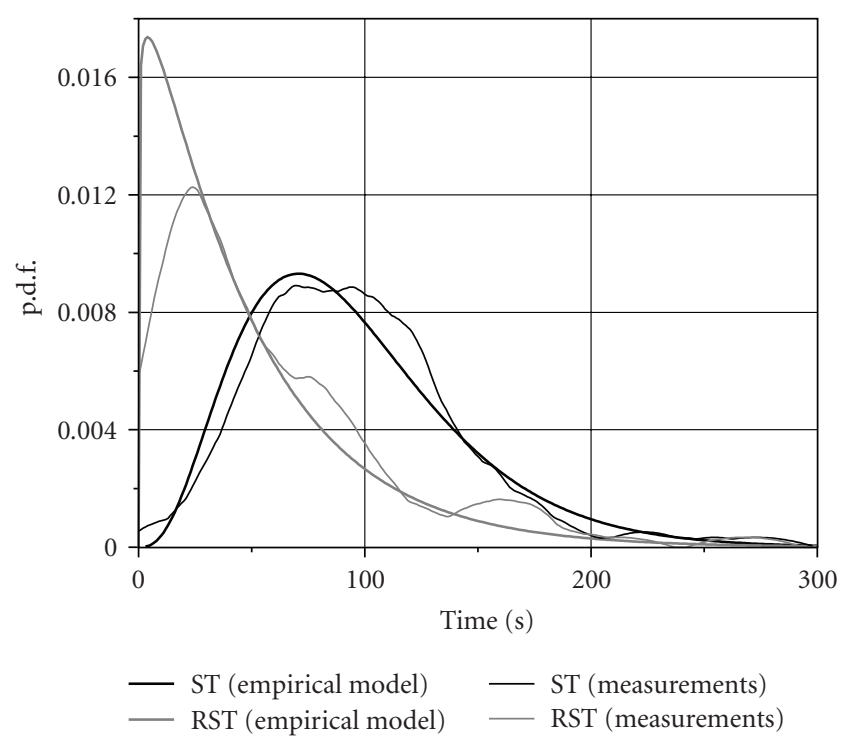

Figure 8: Comparison of the proposed model and measurements for the cell of Figure 7. The values used for the model are $a^{\mathrm{ST}}=$ $3.88094, b^{\mathrm{ST}}=24.5184, a^{\mathrm{RST}}=1.08972$, and $b^{\mathrm{RST}}=44.6443$.

it is evident that the model conformance to measurements is improved, in comparison to [9]. This is due to the use of conditional probabilities of turning, the use of $R$ parameter, and better fitting method.

\section{Conclusion}

A deterministic simulation model is proposed. The model can be used along with a road map in order to provide sojourn time and remaining sojourn time statistics for an arbitrary city cell with low vehicle traffic. The calculation of the parameter $p_{\mathrm{tr}}$ is described in detail. The model has been tested in a real cell, and the results show a satisfactory measurement conformance. This model can be used for cell planning and handover ratio estimation in any urban circular cell that has a rectangular block shape.

\section{References}

[1] D. Hong and S. S. Rappaport, "Traffic model and performance analysis for cellular mobile radio telephone systems with prioritized and nonprioritized handoff procedures," IEEE Transactions on Vehicular Technology, vol. 35, no. 3, pp. 77-92, 1986.

[2] R. A. Guerin, "Channel occupancy time distribution in a cellular radio system," IEEE Transactions on Vehicular Technology, vol. 36, no. 3, pp. 89-99, 1987.

[3] P. I. Bratanov and E. Bonek, "Mobility model of vehicle-borne terminals in urban cellular systems," IEEE Transactions on Vehicular Technology, vol. 52, no. 4, pp. 947-952, 2003.

[4] E. Vagenas, G. S. Paschos, and S. A. Kotsopoulos, "An analytical study of remaining sojourn time in cellular mobile networks," to appear in IEEE Transactions on Vehicular Technology.

[5] M. Benveniste, "Probability models of microcell coverage," Wireless Networks, vol. 2, no. 4, pp. 289-296, 1996.
[6] M. M. Zonoozi, P. Dassanayake, and M. Faulkner, "Teletraffic modeling of cellular mobile networks," in Proceedings of the 46th IEEE Vehicular Technology Conference (VTC '96), vol. 2, pp. 1274-1277, Atlanta, Ga, USA, May 1996.

[7] I. Seskar, S. V. Maric, J. Holtzman, and J. Wasserman, "Rate of location area updates in cellular systems," in Proceedings of the 42nd IEEE Vehicular Technology Conference (VTC '92), vol. 2, pp. 694-697, Denver, Colo, USA, May 1992.

[8] F. Barcelo and J. Jordan, "Channel holding time distribution in public telephony systems (PAMR and PCS)," IEEE Transactions on Vehicular Technology, vol. 49, no. 5, pp. 1615-1625, 2000.

[9] G. S. Paschos, E. Vagenas, and S. A. Kotsopoulos, "User mobility model based on street pattern," in Proceedings of the 61st IEEE Vehicular Technology Conference (VTC '05), vol. 4, pp. 2123-2126, Stockholm, Sweden, May-June 2005.

[10] I. G. Tsoulos, D. Gavrilis, and E. Dermatas, "GDF: a tool for function estimation through grammatical evolution," Computer Physics Communications, vol. 174, no. 7, pp. 555559, 2006.

[11] D. W. Scott, Multivariate Density Estimation, Theory, Practice and Visualization, John Wiley \& Sons, New York, NY, USA, 1992. 

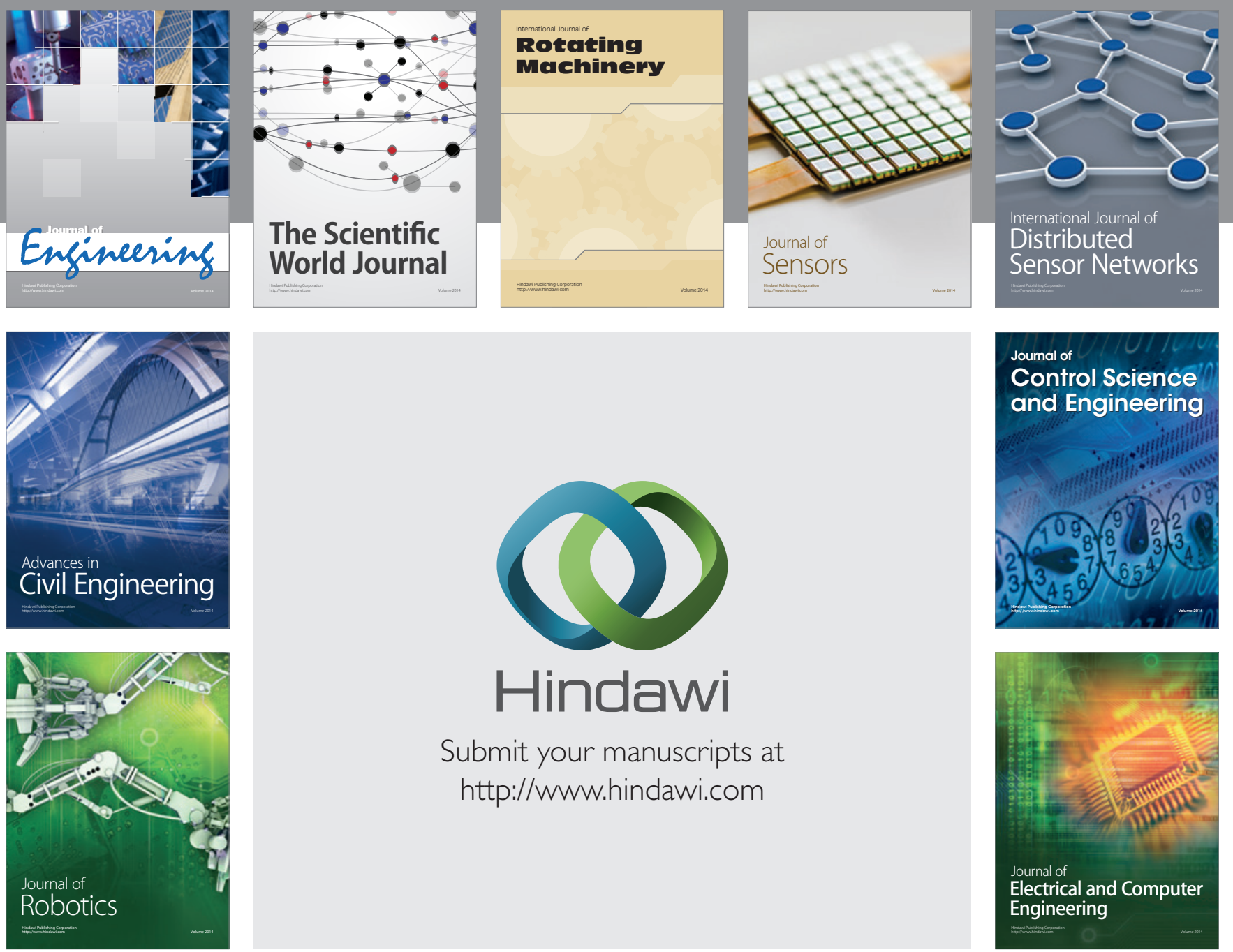

Submit your manuscripts at

http://www.hindawi.com
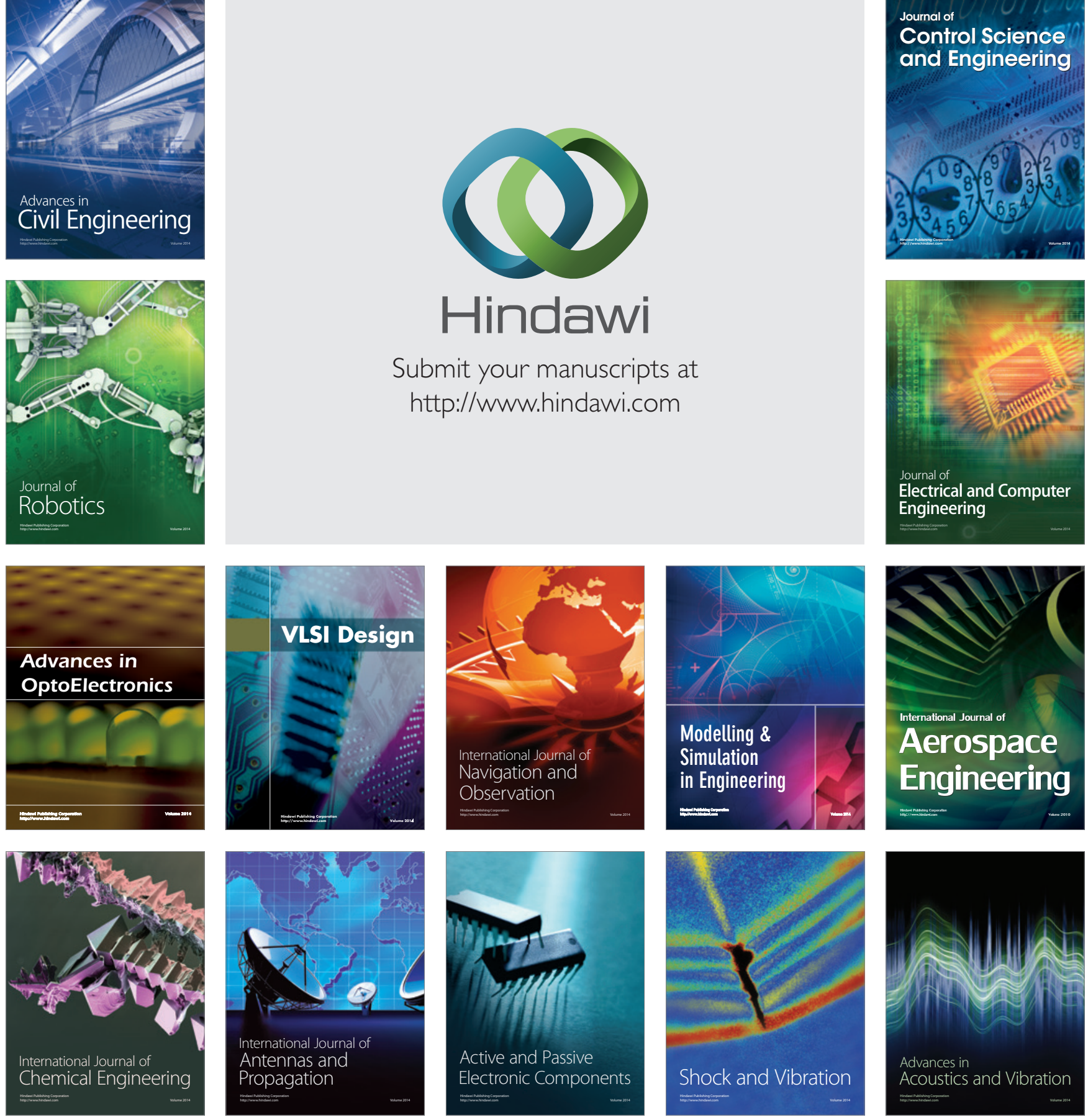